\title{
Jamming and force distribution in growing epithelial tissue
}

\author{
Pranav Madhikar $\odot,{ }^{1}$ Jan Åström $\odot,{ }^{2}$ Björn Baumeier $\odot,{ }^{1}$ and Mikko Karttunen $\odot^{3,4,5, *}$ \\ ${ }^{1}$ Department of Mathematics and Computer Science \& Institute for Complex Molecular Systems, Eindhoven University of Technology, \\ 5600 MB, Eindhoven, The Netherlands \\ ${ }^{2}$ CSC Scientific Computing Ltd, Kägelstranden 14, 02150 Esbo, Finland \\ ${ }^{3}$ Department of Chemistry, The University of Western Ontario, 1151 Richmond Street, London, Ontario, Canada N6A 5B7 \\ ${ }^{4}$ Department of Applied Mathematics, The University of Western Ontario, 1151 Richmond Street, London, Ontario, Canada N6A 5B7 \\ ${ }^{5}$ The Centre for Advanced Materials and Biomaterials Research, The University of Western Ontario, \\ 1151 Richmond Street, London, Ontario, Canada N6A 3 K7
}

(Received 5 May 2020; accepted 15 April 2021; published 19 May 2021)

\begin{abstract}
We investigate morphologies of proliferating cellular tissues using a numerical simulation model for mechanical cell division and migration in two dimensions. The model is applied to a bimodal mixture consisting of stiff cells with a low growth potential and soft cells with a high growth potential; cancer cells are typically considered to be softer than healthy cells. In an even mixture, the soft cells develop into a tissue matrix and the stiff cells into a dendritelike network structure. When soft cells are placed inside a tissue consisting of stiff cells (to model cancer growth), the soft cells develop into a fast-growing tumorlike structure that gradually evacuates the stiff cell matrix. The model also demonstrates (1) how soft cells orient themselves in the direction of the largest effective stiffness as predicted by the theory of Bischofs and Schwarz [Proc. Natl. Acad. Sci. USA 100, 9274 (2003)] and (2) that the orientation and force generation continue a few cell rows behind the soft-stiff interface. With increasing intercell friction, tumor growth slows down, and cell death occurs. The contact force distribution between cells is demonstrated to be highly sensitive to cell type mixtures and cell-cell interactions, which indicates that local mechanical forces can be useful as a regulator of tissue formation. The results shed light on established experimental data.
\end{abstract}

DOI: 10.1103/PhysRevResearch.3.023129

\section{INTRODUCTION}

Morphology and dynamics of proliferating cells are fundamental issues in cellular development [1-6]. They are controlled by a number of factors, but from the physical point of view, morphology is tightly coupled to inter-cellular force transmission, see, e.g., Refs. [6-8]. Mechanical forces have been shown to be important in tissue healing after damage [9] and cancer development, and it has been suggested that tumor growth may even become arrested by intercellular mechanical forces [10-12]. In addition to uniform structures, a plethora of structures with various mechanisms and division modes have been suggested, but the issue remains largely unresolved [13-16].

Among the many complications in investigating force transmission are that, at their embryonic state, cells may not yet have developed junctions and may display more fluidlike behavior, and cell-cell adhesion depends on the cell type and type of adhesion (focal or nonfocal) $[7,15,17,18]$. Junctions are crucial in cell-to-cell stress transmission $[7,8,19,20]$, but

\footnotetext{
*mkarttu@uwo.ca

Published by the American Physical Society under the terms of the Creative Commons Attribution 4.0 International license. Further distribution of this work must maintain attribution to the author(s) and the published article's title, journal citation, and DOI.
}

it is challenging to probe the individual junctions experimentally. In addition, the different cellular level signaling mechanism can be coupled depending on local properties and environment; that is, chemical signaling based on molecular interactions and mechanotransduction may depend on each other, see, e.g., Ref. [21].

From a coarse-grained point of view, i.e., ignoring chemical details and treating cells as elastic objects, cellular systems can be seen as disperse grand canonical soft colloidal systems under evolving pressure. Several studies have tried to capture aspects of growing soft matter systems [22-25], but even in simple systems, many fundamental questions remain open, including the precise nature of colloidal phase diagrams when colloids are soft with size dispersity [26] and structure selection via self-assembly [27]. Cellular systems are even more complex since they exhibit additional behaviors such as growth and division, they have varying mechanical properties (e.g., elasticity and cell-cell adhesion), and their responses to external stimuli may be sensitive to the local environment.

Dimensionality has an important role in regulation of intracellular and intercellular forces at different levels, see, e.g., Refs. [28-32]. Systems such as epithelial tissues and Drosophila wing discs are inherently two dimensional (2D), which gives them distinct morphological properties due to the nature of cellular packing and transmission of and response to forces $[5,15,33]$. In addition, jamming can be very strong in 2D; understanding the effects of stiffness, density, and intermembrane friction is crucial for determining how 


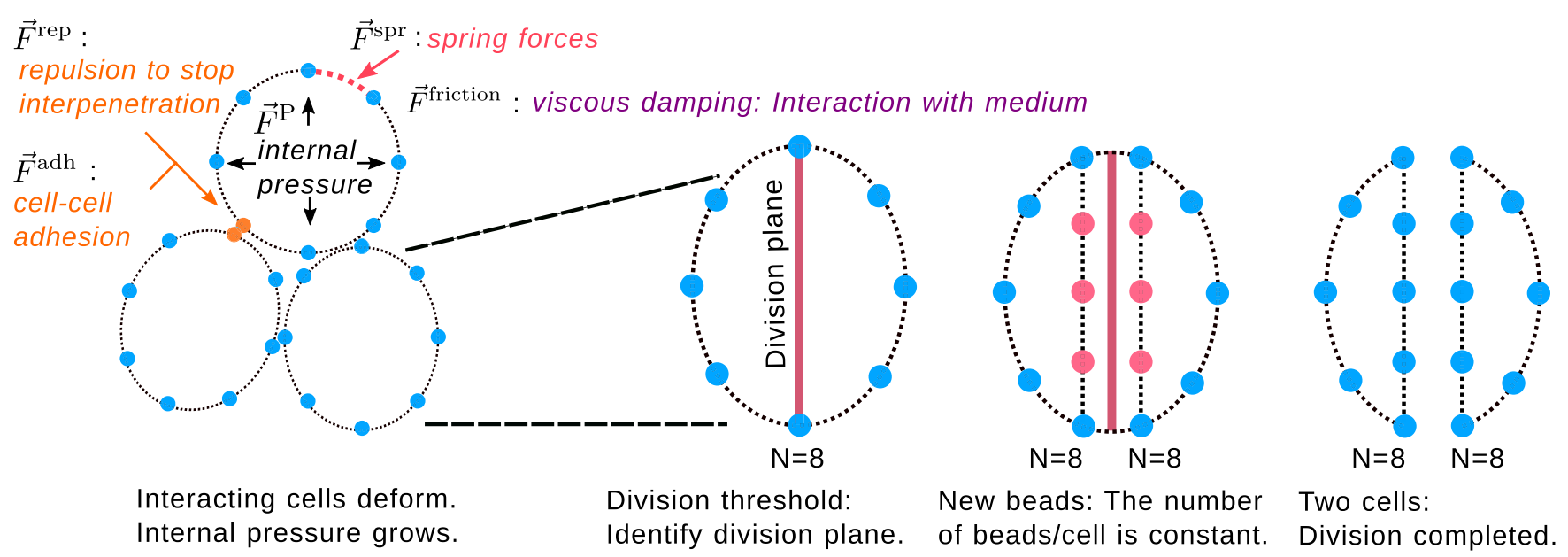

FIG. 1. Illustration of the model and the forces [Eq. (1)]. As the cells grow and deform, they reach the division threshold. At that time, a division plane is identified based on the long axis (Hertwig's) or some other selected rule. As the cell divides, new beads must be added to preserve the topology of the cells. Once division is completed, two child cells are produced. Colors indicate the different forces and the new beads that need to be created at the time of division.

jamming emerges in cellular systems [34,35]. Besides being important in understanding the mechanisms of cell movement under pressure [8], such situations have been proposed to be important in tumor growth [10,11]-cancer cells are often softer than healthy cells [36], although the opposite has also been reported [37]. Our main focus is on the above effects in systems consisting of hard cells in a soft matrix and vice versa. We use our previously introduced 2D computational model called EPICELL2D (Epithelial Cell 2D) [38]. The model is summarized below in the Models and Methods section and described in full detail in Ref. [38]. Cell stiffness, its measurements, and connection to cancer metastasis have been recently reviewed by Luo et al. [39].

2D cellular systems have been studied with a number of computational methods including off-lattice vertex models [14,40-49] and Voronoi tessellation or Delaunay triangulation-based models [50-54]. Such models approximate cell membranes as edges or planes, and they do not include intercellular forces. The immersed boundary method of Rejniak [55], which explicitly models intermembrane interactions is better suited to problems that require intercellular forces. Other methods include lattice-based methods such as the cellular Potts model [56-59]. Although versatile, they describe cellular interactions with scalar energy terms, making it impossible to study forces between cells unless they are amended. Phase-field modeling, based on defining a free energy functional and an order parameter (or several parameters) is an approach to cell division and migration [60-63].

The models that describe cells as discrete entities with individual properties can be further classified as either offlattice [64] or lattice-based [65] agent models. For example, the former include the models of Rejniak [55], Rejniak and Anderson [66], and our current model, and the latter cellular automata and Potts models. In off-lattice models, cells are typically deformable, and forces or energies are used in determining their behavior, whereas in lattice-based models, updates are done based on predetermined rules rather than forces, and the cellular shapes or topologies are typically fixed. Both approaches have their advantages and caveats.
A comprehensive review of agent-based cellular models is provided by Van Liedekerke et al. [67], and a review of mechanobiological aspects and morphology during the whole cell cycle is provided by Clark and Paluch [68].

\section{MODEL AND METHODS}

We employ the EPICELL2D model to study tissue morphologies. Full details, derivation, and parameter mapping with all parameters (force field) are provided in Ref. [38] and not repeated here. Below, we summarize the salient features and provide the new additions. In the context of the models discussed above, EPICELL2D can be classified as an off-lattice agent-based model; cells are well-defined entities, have individual properties, can deform and divide, and forces are used to update their positions and motions.

In EPICELL2D, the cell membrane is discretized as beads connected by elastic bonds of stiffness $K_{i}^{\mathrm{spr}}$ to form a closed loop, see Fig. 1. In our simulations, the cells had 76 beads (Fig. 1 has only eight for clarity). The model has been previously tested [38] using 10-100 beads per cell, and it was found that the number of beads has no effect on results if $N \geqslant 40$. The model parameters (details in Ref. [38]) were mapped using a cell diameter of $10 \mu \mathrm{m}$, mass of $10^{-12} \mathrm{~kg}$ [69], and Young's modulus from mitotic HeLa cells [70]. The orientation of the division plane was chosen randomly such that it passed through the center of mass of the cell.

In EPICELL2D, the force field is defined as a sum of intracellular, intercellular, and cell-medium interactions, see Fig. 1. The intracellular terms include the internal pressure $\left(\vec{F}^{\mathrm{P}}\right)$ and spring forces $\left(\vec{F}^{\mathrm{spr}}\right)$, which provide a mean-field description for the components that give cells their integrity (cell cortex contractility), that is, the cell membrane and cytoskeleton. The intercellular terms define the interactions between the cells. The first of them $\left(\vec{F}^{\text {rep }}\right)$ is repulsion to prevent cells from penetrating each other, and the second term $\left(\vec{F}^{\text {adh }}\right)$ describes cell-cell adhesion. In real cells, adhesion occurs, e.g., due to lipids and different adhesive proteins depending on the cell type. For a recent discussion on the physical aspects of 
adhesion, see, e.g., Schulter et al. [71] and van Helvert $e$ t al. [18]. The final term is medium-cell interaction (friction). With these, the force field can be given in the following general form:

$$
\vec{F}=m \ddot{\vec{r}}=\underbrace{\vec{F}^{\mathrm{P}}+\underbrace{\vec{F}^{\mathrm{spr}}}_{\text {bonded }}}_{\text {intracellular }}+\underbrace{\vec{F}^{\mathrm{rep}}+\vec{F}^{\text {adh }}}_{\text {cell-cell }}+\underbrace{\vec{F}^{\text {friction }}}_{\text {cell-medium }} .
$$

The functional forms of the terms and all the parameters (14 in total) are given in Mkrtchyan et al. [38] and are not repeated here.

In EPICELL2D, cellular growth is controlled by a growth pressure. This is motivated by the fact that cells have mechanisms to control their internal hydrostatic pressure particularly before division [70]. For simplicity, we use a constant, timeindependent pressure for all cells except for a short initial ramp-up phase in pressure. Division is triggered by a threshold in cell area (above which cells divide). We would also like to note that different criteria can be used. As pointed out by Streichan et al. [72], at least area and growth rate are possible criteria for triggering cell division. Daughter cells inherit the pressure from the parent cell, which means they can grow to the same size as the parent until crowding begins to limit growth.

Importantly, EPICELL2D allows the aggregate topology (the polygonal distribution) to vary spontaneously [38]; note that cellular topology is fixed, that is, the number of nodes per cell must remain constant upon division, Fig. 1.

One important issue in modeling cells is cell death. The role of cell death in tissue dynamics has been discussed in numerous publications; a recent perspective is given by Green [73]. Different approaches have been taken to include cell death in computational models. In some models, that is done as a probability for a cell to disappear [74] or via a tunable cell cycle rate [75]. In this paper, cell death occurs due to local stresses. All of these approaches can be justified, as cell death is a very complex and multifaceted phenomenon. Our choice is based on the observations of Chen [76] and Streichan et al. [72] that mechanical constraints, specifically cell shape and local stress, are critical factors and determinants for cell cycle and death. Similar mechanisms have also been proposed and analyzed by Shraiman [77].

Previously, we focused on model development, parameter mapping, and verification against experiments using only one type of cells [38]. Due to the large parameter space (14 in total), it is not feasible, however, to attempt to map a phase diagram. We now extend EPICELL2D for simulations of different cell types using two simple approaches with parameters based on experiments:

noitemsep changing cell stiffness, and

noiitemsep changing the cell-cell friction between cell membranes; in EPICELL2D, cell membrane and cytoskeleton are treated as a coarse-grained single object.

Modification (1) allows for simulations of different cell types. As mentioned above, cancer cells are typically softer than the matrix cells, and softness or higher malleability is typically associated with the invasiveness of cancer cells [36,39]. This has recently been challenged by Nguyen et al. [78], who measured Young's modulus of pancreatic cancer cells using different cell lines and found the stiffer (than the matrix cells) cells to be more invasive than the softer cancer cells. Whether this is purely mechanical or due to simultaneously occurring biological processes remains unclear; simulations using EPICELL2D indicate that stiffer cells migrate easier following in the wake of a leader, while softer cells collectively evacuate stiffer cells due to aggressive growth. This is in excellent agreement with the findings of Trepat et al. [9] who showed that collective effects are essential in cell migration. We will return to this issue in the Results as well as in the Discussion sections.

Here, we use two types of cells: (i) Stiff cells with a low growth potential with stiffness $K_{1}^{\text {spr }}=4 \mu \mathrm{N} / \mu \mathrm{m}$. The low growth potential means that the cell membrane is so stiff that the constant pressure reached after the ramp-up phase is barely enough to grow the cell to a size above the division threshold. (ii) Soft cells with stiffness $K_{2}^{\mathrm{spr}}=1 \mu \mathrm{N} / \mu \mathrm{m}$. These cells have a high growth potential, which means that cell membrane stiffness is low, and the cell area can easily grow beyond the division size; due to the lower elastic modulus, the growth rate of a soft cell is higher even if the internal pressure is the same as for a stiff cell. Note also that, in EPICELL2D, the plasma membrane and cytoskeleton are treated as a coarse-grained single object referred to as the membrane.

Modification (2) allows for comparisons of systems of cells with different intermembrane friction coefficients [term $\vec{F}$ adh in Eq. (1) and Fig. 1]. The importance of cell-cell friction in mechanotransduction has recently been reviewed by Angelini et al. [79].

In Eq. (1), intermembrane friction is modeled via the term $-\mu \vec{v}_{i j}$, where $\mu$ is the friction coefficient, and $\vec{v}_{i j}$ is the relative velocity between two membranes tangential to the cell that the bead $i$ belongs to. We compare systems with $\mu=0.0 \mu \mathrm{g} / \mathrm{s}$ - that is, cells do not interact very much with their neighbors - and strongly interacting cells with $\mu=$ $200.0 \mu \mathrm{g} / \mathrm{s}$. In the simulations, both open and closed boundaries were used.

\section{RESULTS}

We focus on systems with two cell types, stiff and soft, present simultaneously. Soft cells represent tumor cells based on the fact that cancer cells tend to be softer [36]; Young's modulus for cancer cells is typically $\approx 0.5 \mathrm{kPa}$, whereas for normal cells, it often $\approx 1.0-2.0 \mathrm{kPa}$ (although variations are large, see, e.g., Ref. [18]). In real cancer cells, this effect can be enhanced by a lesser number (or lack) of adhesion proteins. However, although cancer cells are generally assumed to grow faster than healthy cells, measurements are not trivial, as different metrics can be used [80]. An additional complication is the fact that, in healthy tissue, growth is regulated, whereas cancer cells typically lack such regulation. For a review of properties of cancer cells, see, e.g., Hanahan and Weinberg [81] and for biomechanical aspects Fritsch et al. [82].

The initial setups were created by placing equal proportions of stiff (red) and soft (blue) cells randomly, see Fig. 2(a); the simulations were repeated several times, and the results did not depend on the initial conditions. Initially, at low coverage, all cells grow to the size at which they divide. At higher coverage, however, growth becomes restricted by 

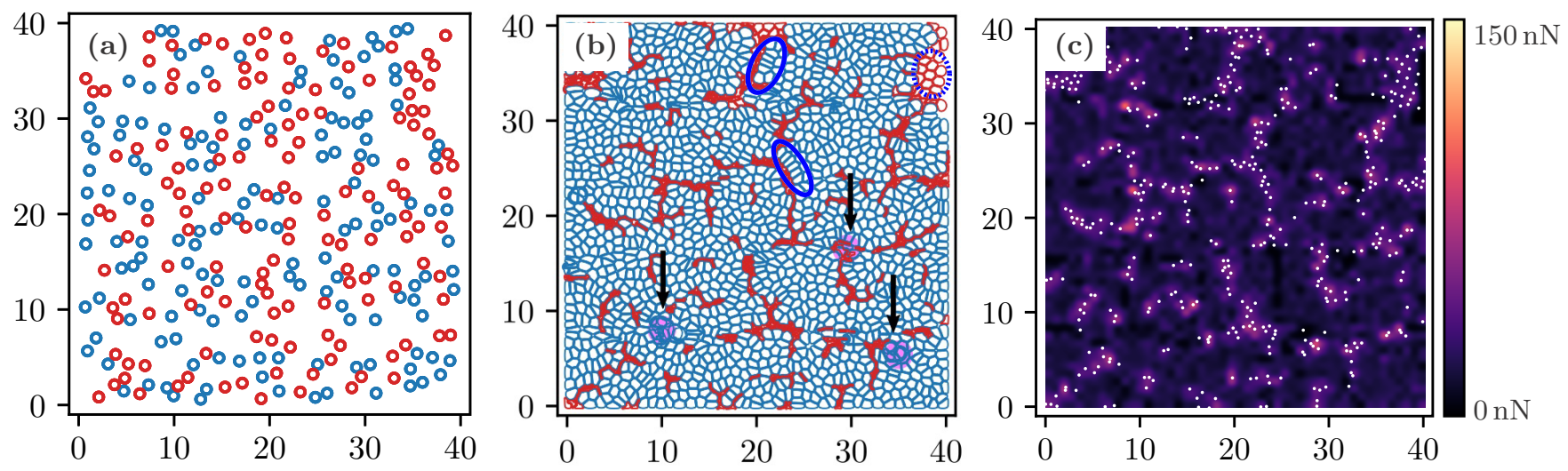

FIG. 2. Influence of stiffness differences in mixtures of stiff (red) and soft (blue) cells. The $X$ and $Y$ axes represent spatial coordinates in two dimensions (2D) in units of $10 \mu \mathrm{m}$. (a) Initial configuration. Growth is simulated from this state onward until confluence with intermembrane friction coefficient $\mu=0.0 \mu \mathrm{g} / \mathrm{s}$. (b) A confluent tissue of soft and stiff cells. Stiff cells form dendrite or veinlike structures in a matrix of soft cells. The regions marked with light purple and arrows are areas where cells interpenetrate and cell death occurs. The areas indicated with blue ovals show how the soft cells orient themselves at the boundary of stiff cells. The area surrounded by an oval with dashed lines shows how the stiff cells retain their shapes when away from the boundary. These issues are elaborated in the Discussion section. (c) Contact force distribution in the same tissue. Large contact forces are located at stiff cells and at boundaries between soft and stiff cells. White markers are the centers of masses of the stiff cells.

lack of space $[38,83]$. The soft cells reach division size much earlier and therefore divide about 10 times faster than stiff cells. Figure 2(b) shows the tissue structure at the end of one of the simulations. The soft cancer cells have invaded the space, while the stiff cells (red) have been compressed into dendritelike structures. Another distinct feature is that the cells interpenetrate in the regions marked with light purple and arrows in Fig. 2(b). Although this behavior may seem as an artifact, it occurs in diverse systems, as has been shown by Eisenhoffer et al. [84] for canine, human, and zebrafish epithelial cells and discussed at length by Guillot and Lecuit [15] (see fig. 2 in Ref. [15]). Figure 2(c) shows the average contact forces between cells. The white dots show the centers of masses of the stiff cells. The contact force peaks correlate highly with the locations of the stiff cells indicating that the soft cells overwhelm the stiff cells as the tissue grows. In similar simulations with only one type of cell, the contact forces are much more homogeneous at similar degree of coverage.

At these interpenetrating cells is where cell death occurs. Although the dead cells are not physically removed (to keep the simulation code faster), they collapse and occupy only minimal space as defined by the nonoverlap of the surface nodes.

Next, we examine if the collapse of stiff cells can be mitigated by making their interactions stronger. This is done by changing the magnitude of intermembrane friction $\mu$. Since cells need space to grow, they need to slide past each other into empty regions. Higher friction, however, induces jamming, and thus, reaching the division threshold takes a much longer time. The softer cells will also need to counteract this effect to be able to grow.

Figure 3 shows a similar simulation setup as before, except with two different values of friction $\mu$. Figure 3(a) shows the initial conditions and Figs. 3(b) and 3(c) the final states for $\mu=0.0 \mu \mathrm{g} / \mathrm{s}$ and $\mu=200.0 \mu \mathrm{g} / \mathrm{s}$, respectively. The tissues in Fig. 3(c) grow with open boundaries. In both cases, the simulations were run for a time corresponding to 10 division cycles. As expected, at low intermembrane friction, there are more cells at the end of the simulation indicating faster growth. The high intermembrane friction system is more porous with slower growth. Physically, the frictionless system [Fig. 3(b)] corresponds to very early stages of development when junctions have not yet developed and the latter [Fig. 3(c)] to a case when cell adhesion molecules are present.

To investigate further, we analyzed cellular areas [Fig. 4(a)] and the forces acting on the cells [Fig. 4(b)] at confluence. Both distributions display lower total number of cells in the case of the high-friction tissue. The peak in area distributions is just below $100 \mu \mathrm{m}^{2}$, which is due to the threshold division area $\left(A^{\text {div }}=100 \mu \mathrm{m}^{2}\right)$. Some of the cell areas have grown past this limit as division occurs only at discrete time intervals.

The area distribution for $\mu=0.0 \mu \mathrm{g} / \mathrm{s}$ [Fig. 4(a)] shows a small peak at $A \approx 25 \mu \mathrm{m}^{2}$ due to the higher number of collapsed cells in the low-friction system. The large-area peak represents the soft-cell majority with approximately Gaussian shape. This is consistent with the results from simulations of nondividing soft colloids [22]. For $\mu=200.0 \mu \mathrm{g} / \mathrm{s}$, the distribution develops only a single peak. An approximately Gaussian area distribution for most of the soft cells is an indication of the cell area being governed by local compactification in the same way as occurs for nondividing colloids. It is clear, however, that the amounts of the largest and the smallest cells are strongly affected by the division process, and hence, the area distribution cannot be Gaussian.

The distribution of contact forces can perhaps be best understood by picturing two different phases of tissue: (1) a granular phase in which cell density and tissue structure resemble a granular material near jamming. Then the contact forces have the characteristics of granular force chains with an exponential force distribution at the high force. (2) The other is a densely packed phase in which cells have overcome jamming, cell density approaches space filling, forces have been equilibrated and approach a Gaussian. These aspects are discussed in detail below. 

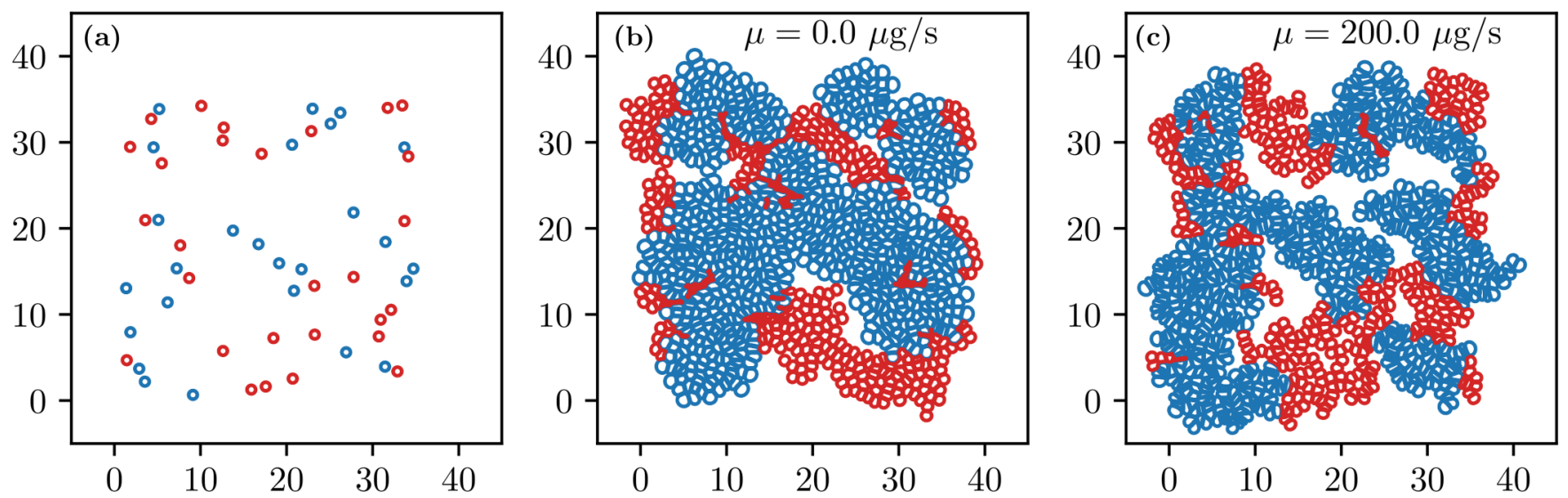

FIG. 3. Influence of intermembrane friction on simulated cell morphologies. The $X$ and $Y$ axes represent spatial coordinates in two dimensions (2D) in units of $10 \mu \mathrm{m}$. (a) Initial conditions used in both (b) and (c). Stiff cells are shown in red and soft ones in blue. The two are present in equal proportions. Morphologies after 10 division cycles in the cases of (b) zero intercellular friction and (c) high friction $(\mu=200.0 \mu \mathrm{g} / \mathrm{s})$. Growth is faster at $\mu=0.0 \mu \mathrm{g} / \mathrm{s}$, as indicated by the larger number of cells after the same number of division cycles. Morphologically, the high intermembrane friction system is more porous. The frictionless system corresponds to very early stages of development when junctions have not yet developed.

Far from confluence, all contact forces would relax toward zero if proliferation stopped and forces were measured after a long enough time. During growth and at low cell-cell friction, the force distribution in Fig. 4(b) is fairly close to a Gaussian (without negative values). There is, however, a hint of two separate peaks; when friction is increased, the distribution deviates more from a Gaussian, and its overall structure approaches an exponential function, indicating that, at zero
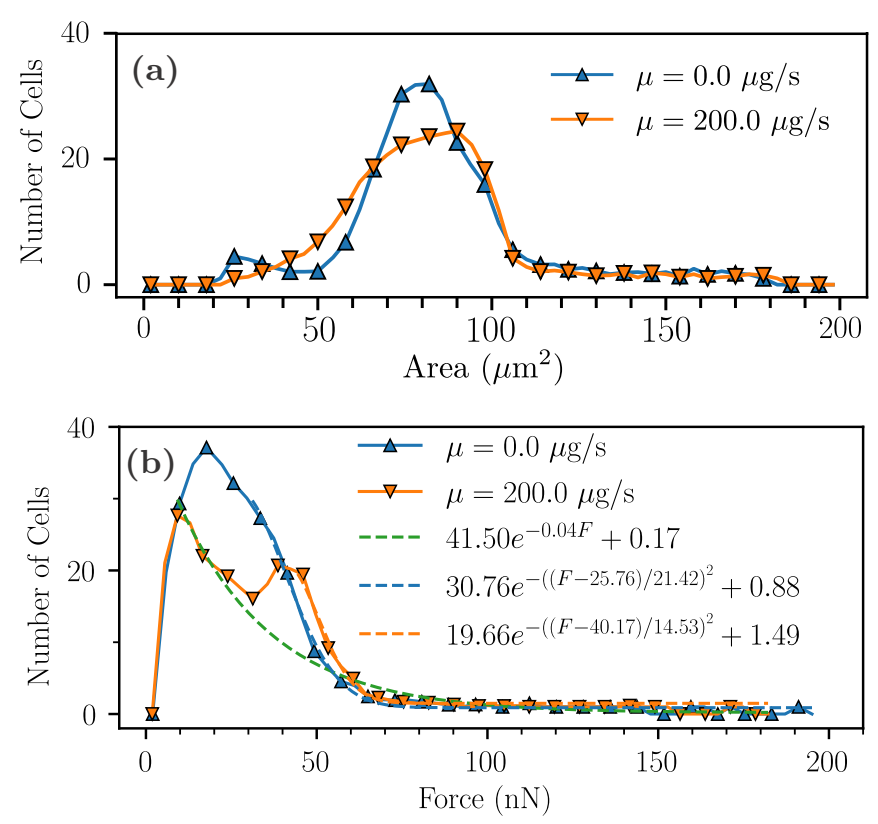

FIG. 4. (a) Area and (b) cell-cell contact force (denoted by $F)$ distributions at low $(\mu=0.0 \mu \mathrm{g} / \mathrm{s})$ and high $(\mu=200.0 \mu \mathrm{g} / \mathrm{s})$ intermembrane friction, corresponding to Figs. 3(b) and 3(c), respectively. (a) The peak at low areas $\left(A \approx 25 \mu \mathrm{m}^{2}\right)$ corresponds to collapsed stiff cells. The dashed lines in (b) are fits with the green line being a fit to the $\mu=200.0 \mu \mathrm{g} / \mathrm{s}$ case, ignoring the second peak. Solid lines are guide to the eye. friction, the tissue is closer to the dense-packed phase, while at higher friction, the tissue is closer to the granular phase. At high friction, the secondary peak in force distribution develops more clearly. Approximately exponential tails have also been reported in the cell cycle experiments of Trepat et al. [9]. We will return to this issue in the Discussion section.

Cells at and above the peaks at larger forces arise as a result of remaining mechanical frustrations particularly at stiff-soft cell boundaries. These frustrations are, as expected, more pronounced at high friction and more relaxed at low friction. Using tissue with only one type of cells present, the high-force tails of the distributions vanish entirely, as there are no such boundaries.

As the final case, we study a cluster of soft cells surrounded by stiff cells to model tumor growth in a healthy tissue and with closed boundary conditions to be able to study densely packed tissues.

Figures 5(a) and 5(b) show morphologies for $\mu=0.0 \mu \mathrm{g} / \mathrm{s}$ and $\mu=200.0 \mu \mathrm{g} / \mathrm{s}$ at confluence. Figure 5 shows that the (a) $\mu=0.0 \mu \mathrm{g} / \mathrm{s}$

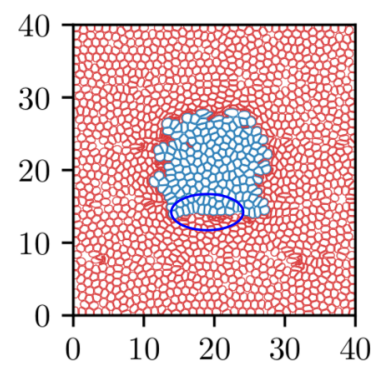

(b) $\mu=200.0 \mu \mathrm{g} / \mathrm{s}$

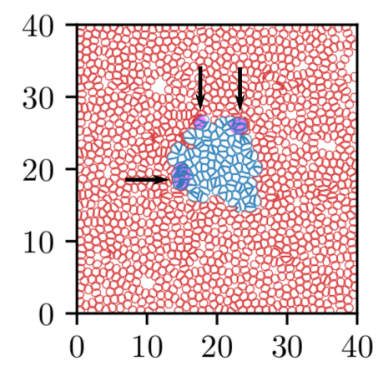

FIG. 5. Configurations at confluence for the case of inclusion of soft (blue) cells in a matrix of stiff (red) cells when (a) $\mu=0.0 \mu \mathrm{g} / \mathrm{s}$ and (b) $\mu=200.0 \mu \mathrm{g} / \mathrm{s}$. The areas indicated with blue ovals show how the soft cells orient themselves at the boundary of stiff cells. Purple regions and arrows: areas were cells interpenetrate and death could occur. $X$ and $Y$ axes: spatial coordinates in units of $10 \mu \mathrm{m}$. 


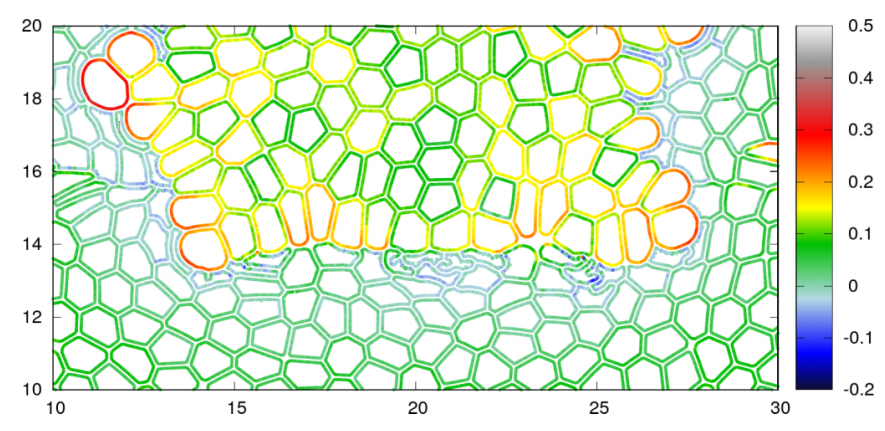

FIG. 6. Close-up showing (roughly) the circled area in Fig. 5(a). The color map shows the strain, that is, relative change in local membrane length as indicated by the color bar (1.0 equals to $100 \%$ strain). Soft cells orient themselves in the direction of the largest effective stiffness (the soft-stiff boundary). Since strain is given as stress/Young's modulus, it is evident that the soft cells at the boundary layer manifest force dipoles as predicted by Bischofs and Schwarz [85].

softer (blue) cells introduced into matrices of stiffer cells grow faster when intermembrane friction is low; weaker cell-cell interactions provide conditions for easier growth. This suggests that intercellular interactions can be an indicator of how well epithelial tissue can damp the growth of rogue cells that have a higher growth potential.

The high-friction system contains a few pores that have formed due to jamming. Cell sizes are roughly equal within the tumor and inside the matrix, but along the tumor boundary, the matrix cells are compressed, and the tumor cells are enlarged. Further analysis also showed that contact forces are lowest far from the tumor, slightly elevated inside, while largest forces are scattered along the tumor boundary. The matrix cells at the boundary are compressed (i.e., they have a negative membrane strain), while the tumor cells are elongated (i.e., they have a positive membrane strain) near the boundary, Fig. 6. This effect is more pronounced in the lowfriction case.

To quantify the above, Figs. 7(a) and 7(b) show the contact force distributions. Independent of friction, the distributions are Gaussian at small forces with an exponential tail. The Gaussian part suggests that the force distribution is uniform. The exponential tail, on the other hand, is a signature of disorder and jamming induced by mechanical frustrations. Here, it results from large differences in the cell-cell contact forces along and near the tumor boundary.

Distributions have also been measured for soft colloidal systems under compression. It is well established that the distribution has an exponential tail in the vicinity of the jamming transition, see, e.g., Refs. [22,86,87] and references therein. Experiments by Jose et al. [87] for three-dimensional packings of soft colloids also show that the distribution well above the jamming transition becomes Gaussian. As Fig. 7 shows, the exponential tail is present at both zero and high friction. The fact that the cells grow also means that their volumes are not conserved (in contrast to typical colloids). This is also the case for the cells that are being pushed and compressed by their neighbors, see the snapshots in Figs. 3 and 5. What is clearly different here is the distribution at low forces: The exponential is preceded by a Gaussian distribution. Gaussian
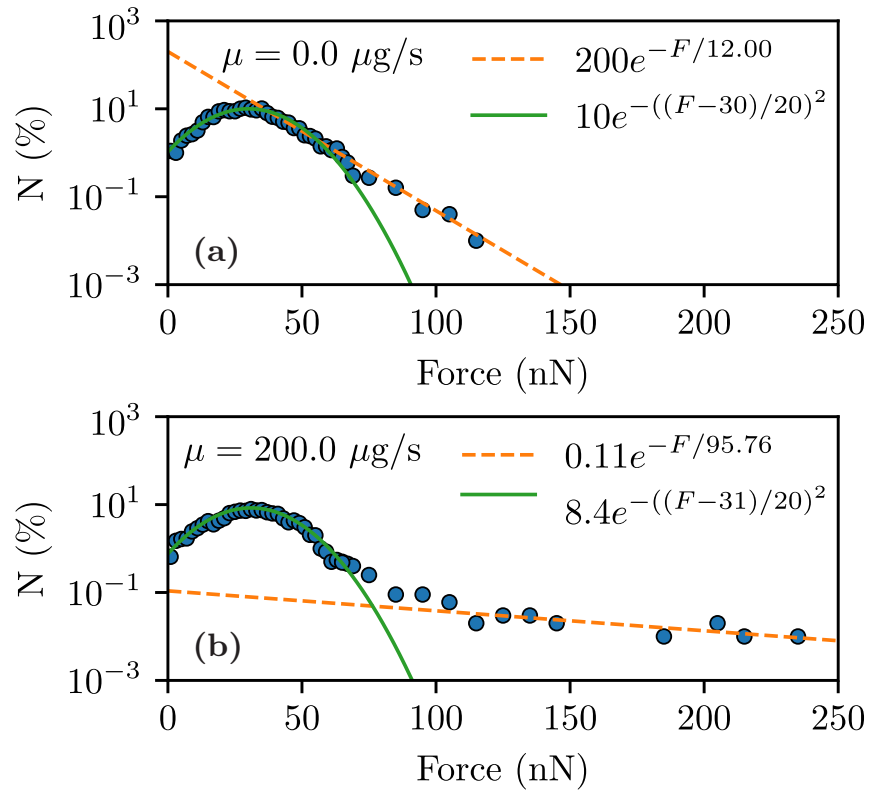

FIG. 7. Distributions of cell-cell contact forces $(F)$ when a soft cell is initially introduced into a tissue of stiff cells. (a) $\mu=0.0 \mu \mathrm{g} / \mathrm{s}$ and (b) $\mu=200.0 \mu \mathrm{g} / \mathrm{s}$. Configurations are shown in Figs. 5(a) and 5 (b), respectively.

peak has been observed in simulations of soft colloids in 2D with zero friction [22].

In contrast, in the three dimensional experiments of Jose et al. [87], the low-force part of the distribution remained almost flat except well above jamming transition. In addition, van Eerd et al. [88] have reported faster-than-exponential decay from their high-accuracy Monte Carlo simulations, although the deviations can be very hard to detect without high-accuracy sampling methods.

\section{DISCUSSION}

Using proliferating Madin-Darby canine kidney cells, Trepat et al. [9] studied collective migration of cells and came to the conclusion that long-range traction forces drive collective migration. They measured traction distributions and found that, in all cases, the distributions had exponential tails. Here, we measured the cell-cell contact forces (Fig. 7) and also found exponential tails. Like Trepat et al. [9], the peak of the distribution appears to be Gaussian. This indicates that our model is capable of capturing the behavior of real systems, and it confirms that mechanical aspects can be modeled using such a coarse-grained approach.

Fritsch et al. [82] point out the conundrum that, although cancers cells are softer than healthy ones, tumors appear as hard lumps. Fritsch et al. [82] write: "At first sight, cell softening is contradictory to the observation that tumours are rigid masses-a notion borne out by the fact that breast tumours are often felt as lumps. Moreover, this apparent softness of tumours would hinder their invasiveness." As Figs. 2 and 5 show, our model is capable of demonstrating the effect of invasive soft tissue, and when growth starts within a matrix, it will become balanced by the pressure from the surrounding tissues (Fig. 5), that is, homeostatic pressure. 
In separate studies, Basan et al. [89] and Podewitz et al. [74] investigated the role of homeostatic pressure using experiments, numerical models, and mesoscale simulations in which cells are represented by two point particles with potentials for growth, adhesion, and exclusion. Constant temperature and pressure ensembles were used for the molecular dynamics simulations [74]. Due to the representation of cells with central potentials, the approach is not capable of modeling cell shape changes to any significant degree. As their main conclusion based on their model and experiments, they showed that negative homeostatic pressure (due to compression in the bulk) is both possible and stable. They also showed that homeostatic pressure increases linearly with increasing cell compressibility. Our results agree with this and also demonstrate that the situation is complex. For example, there is destruction of healthy cells at the boundary as a result of concentrated pressure from the cancer cells. These results indicate why cancer cells may be softer.

Perhaps the most interesting comparison is with the theoretical work of Bischofs and Schwarz [85]. They demonstrated that cells prefer to orient themselves in the direction of the largest effective stiffness. This orientational preference is illustrated in Figs. 2(b) and 5(a) by blue ovals (solid line). The softer blue cells are facing a stiff environment, and as a result, they elongate and organize with their long axis perpendicular to the interface. Figure 6 shows the local strain, which clearly manifests that force dipoles are present at the boundary. This is exactly what is predicted by the theory of Bischofs and Schwarz [85] for clamped (stiff) boundaries. In the interior, the cell shapes are more isotropic, as is also the case with a free boundary, see the oval with the dotted blue line in Fig. 2(b); the outermost cells are not in contact with the boundary yet and hence do not feel it. This is also clearly visible in the simulations with open boundaries, Fig. 2. In addition, Figs. 2(b) and 5(a) show that, when going away from a boundary where the softer cells have elongated, this order may persist for a few cell lines before disappearing, depending on the local environment. Thus, forces are generated away from the immediate boundary. This is in excellent agreement with the experiments of Trepat et al. [9], who argued that force generation is a collective long-range effect rather than requiring "leader cells." The figure also shows that, when the interface between the hard and soft cells has a more complex shape than a straight line, the cellular shapes and their orientations become more complex. This is also visible in the case of high cell-cell friction.

As already discussed in the Introduction, different vertex, Potts, and Voronoi-type models have been used to model cell division. Typically, the focus has been on glasslike behavior in (dense) tissues and topological transitions such as the neighbor exchange T1 transition $[43,44]$, and in some cases, such models have been combined even with dynamics [44,75]. Since the current model gives rise spontaneously to polygonal distributions (as already reported earlier [38]), it would be possible to use it to study topological changes. That is, however, beyond the scope of this paper.

Using a vertex model, Bi et al. [90] demonstrated a new rigidity transition at constant density (confluence). As the key parameter, the model has a (cellular) perimeter-to-area ratio which determines deformability of cells. This parameter has a critical value distinguishing between rigid and fluidlike tissues. The same effect can be observed in our model. Cells with high internal pressure form a rigid tissue, while deflated cells easily change their perimeter area, allowing them to deform and flow past each other. Cell friction also plays a significant role in such behavior. Using a cellular area/rigidity ratio is not an optimal control parameter here, but as Fig. 7 shows, the amount of rigidity is governed by cell-cell friction.

\section{CONCLUSIONS}

We use the EPICELL2D model to study systems of cells of two different types in 2D. Cell populations are differentiated by their membrane-cortex stiffness. We showed that this simple difference is enough, provided internal pressure is identical for both, to favor soft cell growth. Even if a few soft cells are surrounded by stiff cells, it is enough for the softer cells to grow rapidly. This effect can be mitigated by a higher interaction strength between cells. Force distributions show similarities to nonproliferating soft colloidal systems. Although not studied in detail here, the model allows for tuning the cell-cell friction, an issue that has recently been raised by Vinutha and Sastry [91] for shear jamming.

There is one further issue that warrants a comment, namely, relation between the cell growth time after division and the division rate. In general, the coupling of cell growth and division is a complex matter involving various regulation mechanisms, see, e.g., Refs. [92,93]. In the current model, mechanical properties (stiffness) control the growth rate and, consequently, the division rate. There may, however, also be circumstances in which these time scales are independent, as discussed and modeled by Straetmans and Khain [94].

From the modeling perspective, the EPICELL2D approach appears to be very versatile. As discussed in the Introduction and elsewhere in the text, there are several different models, each with their own strengths. EPICELL2D is, however, able to capture a very wide range of phenomena without additional modifications. As we have already shown earlier [38], it can reproduce the polygonal cell distributions and mitotic indices observed experimentally in epithelial systems, and as shown here, force distributions and cellular response to different boundaries agree well with experiments and theory.

\section{ACKNOWLEDGMENTS}

M.K. thanks the Discovery and Canada Research Chairs Programs of the Natural Sciences and Engineering Research Council of Canada (NSERC) for financial support.
[1] E. Paluch and C.-P. Heisenberg, Biology and physics of cell shape changes in development, Curr. Biol. 19, R790 (2009).
[2] Y. Li, H. Naveed, S. Kachalo, L. X. Xu, and J. Liang, Mechanisms of regulating cell topology in proliferating epithelia: 
Impact of division plane, mechanical forces, and cell memory, PLoS One 7, e43108 (2012).

[3] C.-P. Heisenberg and Y. Bellaïche, Forces in tissue morphogenesis and patterning, Cell 153, 948 (2013).

[4] K. Ragkousi and M. C. Gibson, Cell division and the maintenance of epithelial order, J. Cell Biol. 207, 181 (2014).

[5] W. T. Gibson, B. Y. Rubinstein, E. J. Meyer, J. H. Veldhuis, G. Brodland, R. Nagpal, and M. C. Gibson, On the origins of the mitotic shift in proliferating cell layers, Theor. Biol. Med. Model. 11, 26 (2014).

[6] D. Sanchez-Gutierrez, M. Tozluoglu, J. D. Barry, A. Pascual, Y. Mao, and L. M. Escudero, Fundamental physical cellular constraints drive self-organization of tissues, EMBO J. 35, 77 (2015).

[7] M. Rauzi, P. Verant, T. Lecuit, and P.-F. Lenne, Nature and anisotropy of cortical forces orienting Drosophila tissue morphogenesis, Nat. Cell Biol. 10, 1401 (2008).

[8] D. T. Tambe, C. Corey Hardin, T. E. Angelini, K. Rajendran, C. Y. Park, X. Serra-Picamal, E. H. Zhou, M. H. Zaman, J. P. Butler, and D. A. Weitz, Collective cell guidance by cooperative intercellular forces, Nat. Mater. 10, 469 (2011).

[9] X. Trepat, M. R. Wasserman, T. E. Angelini, E. Millet, D. A. Weitz, J. P. Butler, and J. J. Fredberg, Physical forces during collective cell migration, Nat. Phys. 5, 426 (2009).

[10] G. Helmlinger, P. A. Netti, H. C. Lichtenbeld, R. J. Melder, and R. K. Jain, Solid stress inhibits the growth of multicellular tumor spheroids, Nat. Biotech. 15, 778 (1997).

[11] S. Kumar and V. M. Weaver, Mechanics, malignancy, and metastasis: The force journey of a tumor cell, Canc. Metast. Rev. 28, 113 (2009).

[12] K. Alessandri, B. R. Sarangi, V. V. Gurchenkov, B. Sinha, T. R. Kiessling, L. Fetler, F. Rico, S. Scheuring, C. Lamaze, A. Simon, S. Geraldo, D. Vignjevic, H. Domejean, L. Rolland, A. Funfak, J. Bibette, N. Bremond, and P. Nassoy, Cellular capsules as a tool for multicellular spheroid production and for investigating the mechanics of tumor progression in vitro, Proc. Natl. Acad. Sci. USA 110, 14843 (2013).

[13] F. Pilot and T. Lecuit, Compartmentalized morphogenesis in epithelia: From cell to tissue shape, Dev. Dyn. 232, 685 (2005).

[14] M. C. Gibson, A. B. Patel, R. Nagpal, and N. Perrimon, The emergence of geometric order in proliferating metazoan epithelia, Nature (London) 442, 1038 (2006).

[15] C. Guillot and T. Lecuit, Mechanics of epithelial tissue homeostasis and morphogenesis, Science 340, 1185 (2013).

[16] A. Herrero, J. Stavans, and E. Flores, The multicellular nature of filamentous heterocyst-forming cyanobacteria, FEMS Microbiol. Rev. 40, 831 (2016).

[17] T. Lecuit, Adhesion remodeling underlying tissue morphogenesis, Trends Cell Biol. 15, 34 (2005).

[18] S. van Helvert, C. Storm, and P. Friedl, Mechanoreciprocity in cell migration, Nat. Cell Biol. 20, 8 (2017).

[19] Z. Liu, J. L. Tan, D. M. Cohen, M. T. Yang, N. J. Sniadecki, S. A. Ruiz, C. M. Nelson, and C. S. Chen, Mechanical tugging force regulates the size of cell-cell junctions, Proc. Natl. Acad. Sci. USA 107, 9944 (2010).

[20] L.-L. Pontani, I. Jorjadze, V. Viasnoff, and J. Brujic, biomimetic emulsions reveal the effect of mechanical forces on cell-cell adhesion, Proc. Natl. Acad. Sci. USA 109, 9839 (2012).

[21] H. Zhang and M. Labouesse, Signalling through mechanical inputs-a coordinated process, J. Cell Sci. 125, 3039 (2012).
[22] J. A. Åström and M. Karttunen, Cell aggregation: Packing soft grains, Phys. Rev. E 73, 062301 (2006).

[23] G. W. Jones and S. J. Chapman, Modeling growth in biological materials, SIAM Rev. 54, 52 (2012).

[24] D. Gonzalez-Rodriguez, K. Guevorkian, S. Douezan, and F. Brochard-Wyart, Soft matter models of developing tissues and tumors, Science 338, 910 (2012).

[25] J. Kursawe, R. E. Baker, and A. G. Fletcher, Approximate Bayesian computation reveals the importance of repeated measurements for parameterising cell-based models of growing tissues, J. Theor. Biol. 443, 66 (2018).

[26] L. A. Fernández, V. Martín-Mayor, and P. Verrocchio, Phase Diagram of a Polydisperse Soft-Spheres Model for Liquids and Colloids, Phys. Rev. Lett 98, 085702 (2007).

[27] D. Frenkel and D. J. Wales, Designed to yield, Nat. Mat. 10, 410 (2011).

[28] M. Charnley, F. Anderegg, R. Holtackers, M. Textor, and P. Meraldi, Effect of cell shape and dimensionality on spindle orientation and mitotic timing, PLoS ONE 8, e66918 (2013).

[29] V. Hernández-Hernández, D. Rueda, L. Caballero, E. R Alvarez-Buylla, and M. Benítez, Mechanical forces as information: An integrated approach to plant and animal development, Front. Plant Sc. 5, 265 (2014).

[30] A. Lesman, J. Notbohm, D. A. Tirrell, and G. Ravichandran, Contractile forces regulate cell division in three-dimensional environments, J. Cell. Biol. 205, 155 (2014).

[31] M. Osterfield, C. A. Berg, and S. Y. Shvartsman, Epithelial patterning, morphogenesis, and evolution: Drosophila eggshell as a model, Dev. Cell 41, 337 (2017).

[32] L. M. Owen, A. S. Adhikari, M. Patel, P. Grimmer, N. Leijnse, M. C. Kim, J. Notbohm, C. Franck, and A. R. Dunn, A cytoskeletal clutch mediates cellular force transmission in a soft, three-dimensional extracellular matrix, Mol. Biol. Cell 28, 1959 (2017).

[33] W. T. Gibson and M. C. Gibson, Cell topology, geometry, and morphogenesis in proliferating epithelia, Curr. Top. Dev. Biol. 89, 87 (2009).

[34] M. Sadati, N. T. Qazvini, R. Krishnan, C. Y. Park, and J. J. Fredberg, Collective migration and cell jamming, Differentiation 86, 121 (2013).

[35] C. Reichhardt and C. J. Olson Reichhardt, Active matter transport and jamming on disordered landscapes, Phys. Rev. E 90, 012701 (2014).

[36] C. Alibert, B. Goud, and J.-B. Manneville, Are cancer cells really softer than normal cells? Biol. Cell 109, 167 (2017).

[37] S. Suresh, Biomechanics and biophysics of cancer cells, Acta Biomater. 3, 413 (2007).

[38] A. Mkrtchyan, J. Åström, and M. Karttunen, A new model for cell division and migration with spontaneous topology changes, Soft Mat. 10, 4332 (2014).

[39] Q. Luo, D. Kuang, B. Zhang, and G. Song, Cell stiffness determined by atomic force microscopy and its correlation with cell motility, Biochim. Biophys. Acta Gen. Subj. 1860, 1953 (2016).

[40] H. Honda, M. Tanemura, and T. Nagai, A three-dimensional vertex dynamics cell model of space-filling polyhedra simulating cell behavior in a cell aggregate, J. Theor. Biol. 226, 439 (2004).

[41] R. Farhadifar, J.-C. Röper, B. Aigouy, S. Eaton, and F. Jülicher, The influence of cell mechanics, cell-cell interactions, and proliferation on epithelial packing, Curr. Biol. 17, 2095 (2007). 
[42] L. Hufnagel, A. A. Teleman, H. Rouault, S. M. Cohen, and B. I. Shraiman, On the mechanism of wing size determination in fly development, Proc. Natl. Acad. Sci. USA 104, 3835 (2007).

[43] A. G. Fletcher, M. Osterfield, R. E. Baker, and S. Y. Shvartsman, Vertex models of epithelial morphogenesis, Biophys. J. 106, 2291 (2014).

[44] D. M. Sussman, M. Paoluzzi, M. C. Marchetti, and M. L. Manning, Anomalous glassy dynamics in simple models of dense biological tissue, Europhys. Lett. 121, 36001 (2018).

[45] D. B. Staple, R. Farhadifar, J.-C. Röper, B. Aigouy, S. Eaton, and F. Jülicher, Mechanics and remodelling of cell packings in epithelia, Eur. Phys. J. E 33, 117 (2010).

[46] D. A. Fletcher and R. D. Mullins, Cell mechanics and the cytoskeleton, Nature (London) 463, 485 (2010).

[47] R. Nagpal, A. Patel, and M. C. Gibson, Epithelial topology, Bioessays 30, 260 (2008).

[48] A. B. Patel, W. T. Gibson, M. C. Gibson, and R. Nagpal, Modeling and inferring cleavage patterns in proliferating epithelia, PLoS Comput. Biol. 5, e1000412 (2009).

[49] S. Alt, P. Ganguly, and G. Salbreux, Vertex models: From cell mechanics to tissue morphogenesis, Phil. Trans. Roy. Soc. B 372, 20150520 (2017).

[50] G. Schaller and M. Meyer-Hermann, Kinetic and dynamic Delaunay tetrahedralizations in three dimensions, Comput. Phys. Commun. 162, 9 (2004).

[51] G. Schaller and M. Meyer-Hermann, Multicellular tumor spheroid in an off-lattice Voronoi-Delaunay cell model, Phys. Rev. E 71, 051910 (2005).

[52] M. Meyer-Hermann, Delaunay-object-dynamics: Cell mechanics with a 3D kinetic and dynamic weighted Delaunaytriangulation, Curr. Top. Dev. Biol. 81, 373 (2008).

[53] T. Beyer and M. Meyer-Hermann, Multiscale modeling of cell mechanics and tissue organization, IEEE Eng. Med. Biol. Mag. 28, 38 (2009).

[54] M. Radszuweit, M. Block, J. G. Hengstler, E. Schöll, and D. Drasdo, Comparing the growth kinetics of cell populations in two and three dimensions, Phys. Rev. E 79, 051907 (2009).

[55] K. A. Rejniak, An immersed boundary framework for modelling the growth of individual cells: An application to the early tumour development, J. Theor. Biol. 247, 186 (2007).

[56] F. Graner and J. A. Glazier, Simulation of Biological Cell Sorting Using a Two-Dimensional Extended Potts Model, Phys. Rev. Lett. 69, 2013 (1992).

[57] A. Szabó and R. M. H. Merks, Cellular Potts modeling of tumor growth, tumor invasion, and tumor evolution, Front. Oncol. 3, 87 (2013).

[58] A. Shirinifard, J. S. Gens, B. L. Zaitlen, N. J. Popławski, M. Swat, and J. A. Glazier, 3D multi-cell simulation of tumor growth and angiogenesis, PLoS One 4, e7190 (2009).

[59] R. M. H. Merks and J. A. Glazier, A cell-centered approach to developmental biology, Physica A 352, 113 (2005).

[60] M. Nonomura, Study on multicellular systems using a phase field model, PLoS One 7, e33501 (2012).

[61] B. Palmieri, Y. Bresler, D. Wirtz, and M. Grant, Multiple scale model for cell migration in monolayers: Elastic mismatch between cells enhances motility, Sci. Rep. 5, 11745 (2015).

[62] B. Palmieri, C. Scanlon, D. Worroll, M. Grant, and J. Lee, Substrate mediated interaction between pairs of keratocytes:
Multipole traction force models describe their migratory behavior, PLoS One 14, e0212162 (2019).

[63] J. Jiang, K. Garikipati, and S. Rudraraju, A diffuse interface framework for modeling the evolution of multi-cell aggregates as a soft packing problem driven by the growth and division of cells, Bull. Math. Biol. 81, 3282 (2019).

[64] P. van Liedekerke, J. Neitsch, T. Johann, E. Warmt, I. G. Valverde, S. Höhme, S. Grosser, J. Käs, and D. Drasdo, Quantifying the mechanics and growth of cells and tissues in $3 \mathrm{D}$ using high resolution computational models, bioRxiv: https:// doi.org/10.1101/470559.

[65] D. Drasdo, A. Buttenschön, and P. V. Liedekerke, Agent-based lattice models of multicellular systems, in Numerical Methods and Advanced Simulation in Biomechanics and Biological Processes, edited by M. Cerrolaza, S. J. Shefelbine, and D. Garzón-Alvarado (Elsevier, London, UK, 2018), pp. 223-238.

[66] K. A. Rejniak and A. R. A. Anderson, A computational study of the development of epithelial acini: I. sufficient conditions for the formation of a hollow structure, Bull. Math. Biol. 70, 677 (2008).

[67] P. Van Liedekerke, M. M. Palm, N. Jagiella, and D. Drasdo, Simulating tissue mechanics with agent-based models: Concepts, perspectives and some novel results, Comput. Part. Mec. 2, 401 (2015).

[68] A. G. Clark and E. Paluch, Mechanics and regulation of cell shape during the cell cycle, in Cell Cycle in Development. Results and Problems in Cell Differentiation, edited by J. Kubiak (Springer, Berlin, 2011).

[69] K. G. Phillips, S. L. Jacques, and O. J. T. McCarty, Measurement of Single Cell Refractive Index, Dry Mass, Volume, and Density Using a Transillumination Microscope, Phys. Rev. Lett. 109, 118105 (2012).

[70] M. P. Stewart, J. Helenius, Y. Toyoda, S. P. Ramanathan, D. J. Muller, and A. A. Hyman, Hydrostatic pressure and the actomyosin cortex drive mitotic cell rounding, Nature (London) 469, 226 (2011).

[71] D. K. Schluter, I. Ramis-Conde, and M. A. J. Chaplain, Multiscale modelling of the dynamics of cell colonies: Insights into cell-adhesion forces and cancer invasion from in silico simulations, J. Royal Soc. Interf. 12, 20141080 (2014).

[72] S. J. Streichan, C. R. Hoerner, T. Schneidt, D. Holzer, and L. Hufnagel, Spatial constraints control cell proliferation in tissues, Proc. Natl. Acad. Sci. USA 111, 5586 (2014).

[73] D. R. Green, The coming decade of cell death research: Five riddles, Cell 177, 1094 (2019).

[74] N. Podewitz, M. Delarue, and J. Elgeti, Tissue homeostasis: A tensile state, Europhys. Lett. 109, 58005 (2015).

[75] M. Czajkowski, D. M. Sussman, M. C. Marchetti, and M. L. Manning, Glassy dynamics in models of confluent tissue with mitosis and apoptosis, Soft Matter 15, 9133 (2019).

[76] C. S. Chen, Geometric control of cell life and death, Science 276, 1425 (1997).

[77] B. I. Shraiman, Mechanical feedback as a possible regulator of tissue growth, Proc. Natl. Acad. Sci. USA 102, 3318 (2005).

[78] A. V. Nguyen, K. D. Nyberg, M. B. Scott, A. M. Welsh, A. H. Nguyen, N. Wu, S. V. Hohlbauch, N. A. Geisse, E. A. Gibb, A. G. Robertson, T. R. Donahue, and A. C. Rowat, Stiffness of pancreatic cancer cells is associated with increased invasive potential, Integr. Biol. 8, 1232 (2016). 
[79] T. E. Angelini, A. C. Dunn, J. M. Urueña, D. J. Dickrell, D. L. Burris, and W. G. Sawyer, Cell friction, Faraday Disc. 156, 31 (2012).

[80] E. Mehrara, E. Forssell-Aronsson, H. Ahlman, and P. Bernhardt, Specific growth rate versus doubling time for quantitative characterization of tumor growth rate, Cancer Res. 67, 3970 (2007).

[81] D. Hanahan and R. A. Weinberg, Hallmarks of cancer: The next generation, Cell 144, 646 (2011).

[82] A. Fritsch, M. Höckel, T. Kiessling, K. D. Nnetu, F. Wetzel, M. Zink, and J. A. Käs, Are biomechanical changes necessary for tumour progression? Nat. Phys. 6, 730 (2010).

[83] A. Puliafito, L. Hufnagel, P. Neveu, S. Streichan, A. Sigal, D. K. Fygenson, and B. I. Shraiman, Collective and single cell behavior in epithelial contact inhibition, Proc. Natl. Acad. Sci. USA 109, 739 (2012).

[84] G. T. Eisenhoffer, P. D. Loftus, M. Yoshigi, H. Otsuna, C.-B. Chien, P. A. Morcos, and J. Rosenblatt, Crowding induces live cell extrusion to maintain homeostatic cell numbers in epithelia, Nature (London) 484, 546 (2012).

[85] I. B. Bischofs and U. S. Schwarz, Cell organization in soft media due to active mechanosensing, Proc. Natl. Acad. Sci. USA 100, 9274 (2003).

[86] J. M. Erikson, N. W. Mueggenburg, H. M. Jaeger, and S. R. Nagel, Force distributions in three-dimensional com- pressible granular packs, Phys. Rev. E 66, 040301(R) (2002).

[87] J. Jose, A. van Blaaderen, and A. Imhof, Random threedimensional jammed packings of elastic shells acting as force sensors, Phys. Rev. E 93, 062901 (2016).

[88] A. R. T. van Eerd, W. G. Ellenbroek, M. van Hecke, J. H Snoeijer, and T. J. H. Vlugt, Tail of the contact force distribution in static granular materials, Phys. Rev. E 75, 060302(R) (2007).

[89] M. Basan, T. Risler, J.-F. Joanny, X. Sastre-Garau, and J. Prost, Homeostatic competition drives tumor growth and metastasis nucleation, HFSP J. 3, 265 (2009).

[90] D. Bi, J. H. Lopez, J. M. Schwarz, and M. L. Manning, A density-independent rigidity transition in biological tissues, Nat. Phys. 11, 1074 (2015).

[91] H. A. Vinutha and S. Sastry, Disentangling the role of structure and friction in shear jamming, Nat. Phys. 12, 578 (2016).

[92] S. Jun and S. Taheri-Araghi, Cell-size maintenance: Universal strategy revealed, Trends Microbiol. 23, 4 (2015).

[93] G. Facchetti, F. Chang, and M. Howard, Controlling cell size through sizer mechanisms, Curr. Op. Sys. Biol. 5, 86 (2017).

[94] J. Straetmans and E. Khain, Modeling cell size dynamics in a confined nonuniform dense cell culture, J. Stat. Phys. 176, 299 (2019). 